GEOPHYSICAL RESEARCH LETTERS, VOL. 17, NO. 3, PAGES 219-222, MARCH 1990

\title{
PRECISE REGIONAL BASELINE ESTIMATION USING A PRIORI ORBITAL INFORMATION
}

\author{
Ulf J. Lindqwister, Stephen M. Lichten, and Geoffrey Blewitt
}

Jet Propulsion Laboratory, California Institute of Technology

\begin{abstract}
A solution using GPS measurements acquired during the CASA UNO campaign has resulted in 3-4 mm horizontal daily baseline repeatability and $13 \mathrm{~mm}$ vertical repeatability for a $729 \mathrm{~km}$ baseline, located in North America. The agreement with VLBI is at the level of 10-20 $\mathrm{mm}$ for all components. The results were obtained with the GIPSY orbit determination and baseline estimation software and are based on five single-day data arcs spanning the $20,21,25,26$, and 27 of January, 1988. The estimation strategy included resolving the carrier phase integer ambiguities (bias fixing), utilizing an optimal set of fixed reference stations (fiducials), and constraining GPS orbit parameters by applying a priori information (derived from initial multi-day trajectory fits to broadcast ephemeris data). A multi-day (January 20-27) GPS orbit and baseline solution has yielded similar $2-4 \mathrm{~mm}$ horizontal daily repeatabilities for the same baseline, consistent with the constrained single-day arc solutions. The application of weak constraints to the orbital state for single-day data arcs produces solutions which approach the precise orbits obtained with unconstrained multi-day are solutions. This work suggests that the general availability of nominal ephemerides based on many days of global tracking data would be very valuable and practical for regional geodesy, allowing for high precision with single-day network solutions when combined with a continental scale fiducial network.
\end{abstract}

\section{Introduction}

Few millimeter geodetic baseline precision has a broad range of scientific applications, including monitoring strain rates between and within tectonic plates, studying volcanic uplift, and detecting co- and post-seismic net displacements in earthquake fault zones. The CASA UNO experiment offered a unique opportunity to test estimation techniques aimed at achieving such high-precision results, due to the large number of co-monitoring stations with uniform receiver and antenna types. A key requirement for achieving high baseline precision for regional networks is the accurate determination of the GPS satellite orbits. Currently, there are several successful approsches to precise orbit determination based on the fiducial approach, most notably: (1) multi-day arc simultaneous adjustment of all estimated parameters [Dong and Bock, 1989; Lichten and Bertiger, 1989], (2) multi-day arc adjustment where tracking sites are first used to solve for the GPS orbits, followed by a network adjustment of non-tracking sites (i.e., the fixed orbit approach) [Lichten, Bertiger, and Lindqwister, 1989], and (3) single-day arc simultaneous adjustment with carrier phase ambiguity resolution [Blewitt, 1989; Dong and Bock, 1989]. Moreover, combinations of the strategies can be used, e.g. multi-day simultaneous solutions with bias fixing.

This paper investigates the third strategy with the addition of imposing weak a priori constraints on the GPS orbital parameters. The a priori information is obtained from an iterated, 3 week fit to the broadcast ephemeris data, and is therefore independent of the GPS solution set. GPS orbits from weakly constrained and unconstrained solutions are compared to multi-day arc orbits. It is shown that the weakly constrained orbit solutions agree better than the unconstrained solutions with the multi-day results and also show improved baseline repeatabilities. The constrained approach has been widely explored in studies by Beutler and coworkers [Beutler et al., 1987], where orbit constraints are necessary in place of lacking or weak fiducial information. In contrast, our solutions are based on the fiducial reference network (collocated with VLBI) and the constrained orbits simply represent a small but significant enhancement of our solution strategy. The following section discusses the constrained orbit approach, bias fixing, and the choice of fiducial sites. The above strategy with weak orbit constraints has yielded few mm-level horizontal and $10-20 \mathrm{~mm}$ vertical daily repeatabilities for baselines, ranging from 245 to $729 \mathrm{~km}$ in length. In addition, 10-20 mm agreement with VLBI was also obtained for most baseline components. The subsequent section discusses simultaneous multi-day data arc solutions, the most precise GPS orbit strategy. The results are consistent with the constrained single-day solutions. The concluding section summarizes our results and discusses utilizing nominal orbits for regional GPS estimation strategies.

\section{Techniques and Results for Single-Day Estimation}

The single-day arc results discussed below were based on data from six stations in North America: Hat Creek Radio Observatory (CA), Fort Davis (TX), Haystack Observatory (MA), Owens Valley Radio Observatory (CA), Mojave (CA), and Mammoth Lakes (CA). See the map in the first article of this issue (and figure 1) for geographical locations. In order to establish the solutions in a well-defined reference frame and to provide geometrical a priori information, three of the above stations were held fixed at their a priori VLBI locations, for which we used the VIBI global solution entitled: GLB223 (J.W. Ryan, C. Ma, and E. Himwich, Goddard Space Flight Center VLBI group, unpublished results, 1988). See Dong and Bock (1989), Lichten and Border (1987), and references therein for further discussions of the fiducial concept.

Haystack

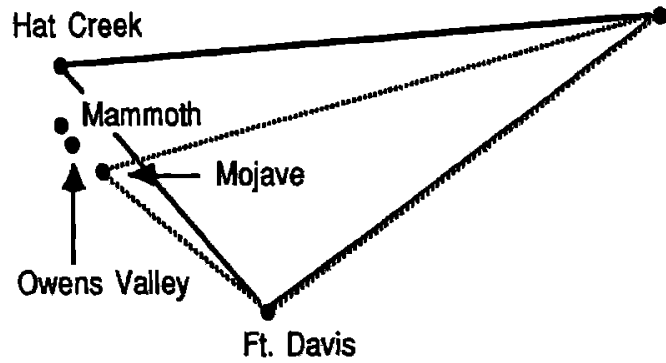

Fig. 1. Two of the three fiducial combinations examined are Hat Creek-Ft. Davis-Haystack (solid lines), and Mojave-Ft. Davis-Haystack (dotted lines). The network with Hat Creek produced $30-50 \%$ better daily baseline repeatabilities.

\section{Optimal Fiducial Geometry}

We have compared the daily repeatability and the agreement with VLBI for baselines estimated with different combinations 
of fiducial sites. For example, utilizing Hat Creek-Ft. DavisHaystack as fiducials rather than Mojave-Ft. Davis-Haystack yields $\sim 30-50 \%$ improvement in daily repeatabilities for the Mojave-Hat Creek (729 km) baseline. The two fiducial combinations are illustrated in figure 1 . The former fiducial network also produces similar improvements in agreement with VLBI for the same baseline. After studying six baselines in California, ranging from 71-729 $\mathrm{km}$ and using three combinations of fixed sites (interchanging Hat Creek with Mojave or Owens Valley), we found that the combination with Hat Creek was superior in all cases. As a rule the most favorable fiducial geometry is obtained when the mobile sites are in proximity to the reference sites and when the spatial extent of the fiducial network is maximized.

During the three week CASA UNO campaign, Hat Creek was only available during 20-22 and 25-27 of January. In addition, only sporadic samples of data were obtained from Mojave on January 22. In order to obtain high-precision solutions a well balanced data set with an optimal fiducial geometry was chosen, i.e., data from five single-day arcs (January 20, 21, and 25-27) were used with Hat Creek, Ft. Davis, and Haystack as fiducial locations. It is somewhat difficult to draw definite conclusions from a statistical sample provided by only five station days. However, we find that by correlating the baseline repeatability results with a study of orbit accuracies, the case for using weak a priori constraints becomes more convincing.

\section{The Standard Estimation Strategy}

The GPS measurements from all sites above were obtained from TI-4100 receivers, with approximately 1.5 meter pseudorange and 1.0 centimeter carrier phase measurement accuracy. The data errors are attributed to multipath and system noise. The TI-4100 receivers track at most four satellites simultaneously. The parameter estimation strategies applied here used 30 second data points compressed to 6 minute normal points acquired at the three fiducials and at the three mobile stations (Owens Valley, Mojave, and Mammoth). On site pressure measurements were used for dry tropospheric delay calibration. The noisier wet tropospheric delay was estimated stochastically in a factorized Kalman filter using a random walk model, applied here to model the time variations in the water vapor induced delay. The random walk model is characterized by the rate of change of the process noise covariance over time and was chosen to be $0.02 \mathrm{~cm} \mathrm{~s}^{-1 / 2}$ corresponding to about $6 \mathrm{~cm}$ delay variation in 24 hours [Lichten and Bertiger, 1989]. The receiver and GPS satellite clocks were estimated as white process noise, i.e. uncorrelated between measurement epochs. The remaining parameters were estimated as constant parameters for the single-day arc solutions. The a priori parameter sigmas are listed in Table 1.

In order to improve the single-day GPS orbit solutions, carrier phase integer biases were resolved. The ambiguity

Table 1. Constraints Used For Parameter Estimation

\begin{tabular}{lll}
\hline & \multicolumn{2}{c}{ A Priori Sigmas } \\
& Constrained & Unconstrained \\
\hline Parameter & $40 \mathrm{~m}$ & $20 \mathrm{~km}$ \\
Satellite position & $2 \times 10^{-3} \mathrm{~m} / \mathrm{s}$ & $20 \mathrm{~m} / \mathrm{s}$ \\
$\begin{array}{l}\text { Satellite velocity } \\
\text { Solar radiation }\end{array}$ & & \\
pressure coefficients & & $25 \%$ \\
$\mathrm{X}, \mathrm{Z}$ & $25 \%$ & $100 \%$ \\
$\mathrm{Y}$ & $100 \%$ &
\end{tabular}

Unconstrained Parameters: Nonfiducial stations, white noise clocks, zenith wet troposphere, and carrier phase biases. Data Weights: carrier phase: $1.0 \mathrm{~cm}$, pseudorange: $1.5 \mathrm{~m}$. resolution technique is based on constraining the carrier phase observables utilizing the precise $\mathbf{P}$ code pseudorange data type [Blewitt, 1989]. The orbital parameters are correlated with the carrier phase ambiguities, hence resolving the ambiguities produces more accurate GPS orbit estimates. Bias fixing in effect converts the carrier phase observable into an ultraprecise pseudorange data type. Differenced carrier biases between all the Califomia stations were resolved for the five single-day arcs. For six Califormia baselines, ranging from 71 $729 \mathrm{~km}$, the daily repeatability and the agreement with VLBI improved by a factor of $\sim 2$ in the east and by $10-20 \%$ in the north and vertical baseline components relative to solutions with unresolved camier biases. In general the longer baselines showed greater improvement since they are more sensitive to GPS orbit errors.

\section{The Constrained Orbit Approach}

In order to achieve precise orbit estimation GIPSY utilizes force modeling which includes the ROCK IV solar radiation pressure model, a gravity field model to degree and order 12 in spherical harmonics, and point masses for the sun, moon, and planets (See Lichten and Border, 1987, and references therein). The orbit integration is performed in the quasi-inertial J2000 reference frame. Orbit estimation proceeds in two steps, where in the first step a nominal satellite trajectory is integrated based on an iterated least-squares fit to a time series of a priori values of the satellite position, velocity, and solar radiation pressure parameters. Broadcast ephemeris data sampled over 3 weeks was used in this study, but in the past we have also used other multi-day fits to global tracking data, for example, from the NSWC precise ephemeris service [Swift, 1985]. The second step is to adjust epoch state values of satellite orbital parameters using GPS carrier phase and pseudorange data types in a factorized Kalman filter. The GIPSY software is flexible in choice of arc length, use of a priori covariances, and stochastic estimation of force and non-force parameters.

The multi-day arc solutions (discussed further in the next section), have resulted in meter-level GPS orbit accuracies [Lichten and Bertiger, 1989]. We have found that our nominal GPS orbits based on 3 week integrated fits to the broadcast ephemeris, when compared with the multi-day arc solutions, proved to be accurate to $\sim 20$ meters with accuracy of about $10^{-7} \mathrm{~cm} / \mathrm{s}^{2}$ for nominal solar radiation pressure coefficients. The broadcast ephemeris is independent of the GPS measurements we used in parameter estimation. In taking advantage of the information in the nominal satellite trajectory, we used fairly conservative a priori sigmas (Table 1) to constrain single-day orbit and baseline estimation. The a prioti sigmas of the satellite positions were chosen to be $40 \mathrm{~m}$ in each component and the sigmas of the $y$-bias solar radiation pressure parameters were $2 \times 10^{-7} \mathrm{~cm} / \mathrm{s}^{2}$ (corresponding to the $100 \%$ value used in the table) [Lichten and Border, 1987].

The effects of using a priori constrained GPS orbits versus unconstrained orbits for single-day arcs are illustrated in figure 2. The mapped estimates from constrained and unconstrained solutions have been differenced from the corresponding estimates obtained in a multi-day arc computation, which here serves as the reference set of orbits. The figure displays mapped differences for the single-day arc 12-hr data interval plus an additional 12-hr prediction interval when no data were taken. The results shown in figure 2 for GPS 8 were similar for all seven satellites: the solution with a priori constraints shows much better agreement with the multi-day arc reference orbit. Note the dramatic improvement over the orbit prediction interval (12-24 hrs) for the constrained orbit case, which is indicative of improved orbit accuracy. For down-track, the GPS 8 formal errors ranged from $0.5-2 \mathrm{~m}, 2.5-5 \mathrm{~m}$, and 7-30 $\mathrm{m}$ for the multi-day arc, constrained single-day arc, and unconstrained single-day arc, respectively. The lower number in each case represents the mms formal error over the 12-hr dam interval and the higher number represents the formal error over 


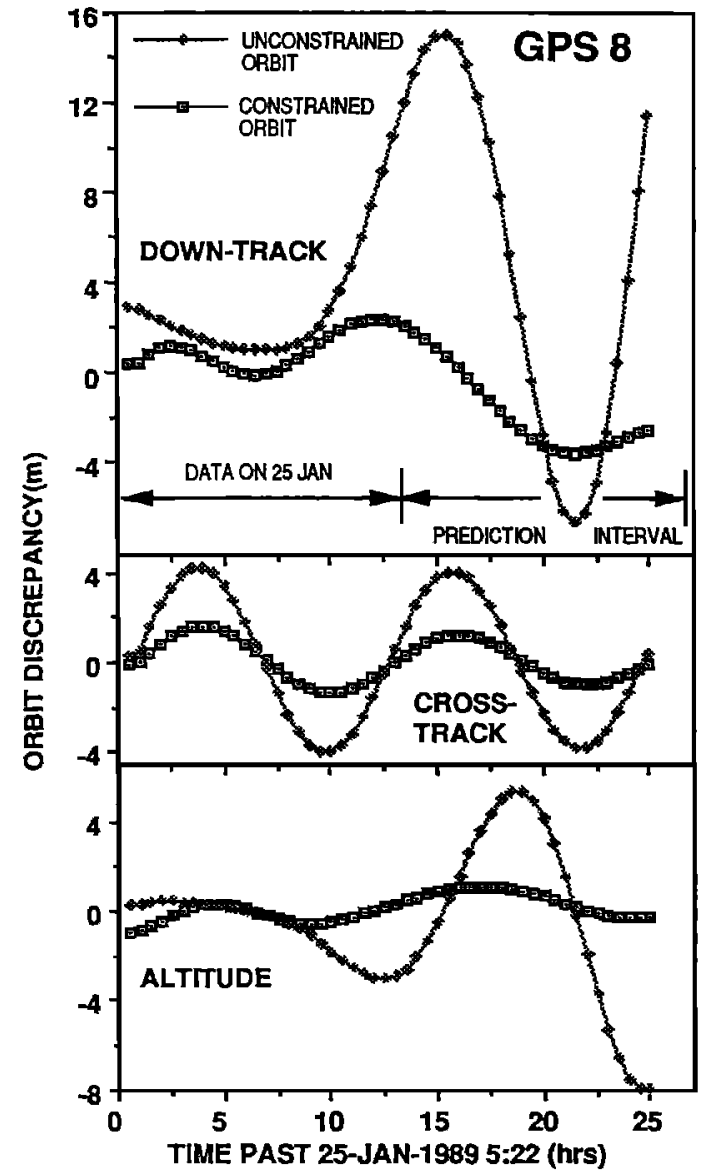

Fig. 2. GPS 8 orbit difference between the reference "truth" orbit determined from a 1-week arc solution, and the constrained and unconstrained single-day arc orbits determined from a 12 -hr pass on January 25 only. The weakly constrained results agree much better with the reference multi-day arc estimates, both in the 12-hr data interval (0-12 hrs) and the 12hr prediction interval (12-24 hrs).

the 12-hr prediction interval (12-hr interpolated interval for the multi-day arc orbit case). Comparison of these numbers to the down-track values plotted at the top of figure 2 shows that the formal errors mirror the actual trends in the data.

In terms of arc length and common view period, GPS 8 was one of the well-tracked satellites in this single-day arc, which included data only from North American tracking sites. However, the formal errors for several satellites with poor visibility stay large (above $10 \mathrm{~m}$ ) over most of this $24-\mathrm{hr}$ period when the single-day orbits are unconstrained. Effectively, only 3-4 satellites play a significant role in baseline parameter estimation for the unconstrained case. By weakly constraining the orbit parameters, however, data from all satellites, including the less visible ones, are used. The covariances stay fairly low (several meters or better) for all the satellites over much of the 24-hr period with constrained orbits. The unconstrained solutions degrade towards the ends of the satellite passes (as do the formal errors), hence the use of constraints effectively increases the length of the observed arc. The key to the dramatic improvement when applying a priori constraints is that the orbital components for different satellites are highly correlated and without such constraints would be dominated by large down-track uncertainties.

In summary, the estimation strategy used for single-day solutions included: fixing carrier phase biases, selecting a strong fiducial geometry, constraining a priori GPS orbits, and utilizing a data set evenly distributed in time for all six stations.

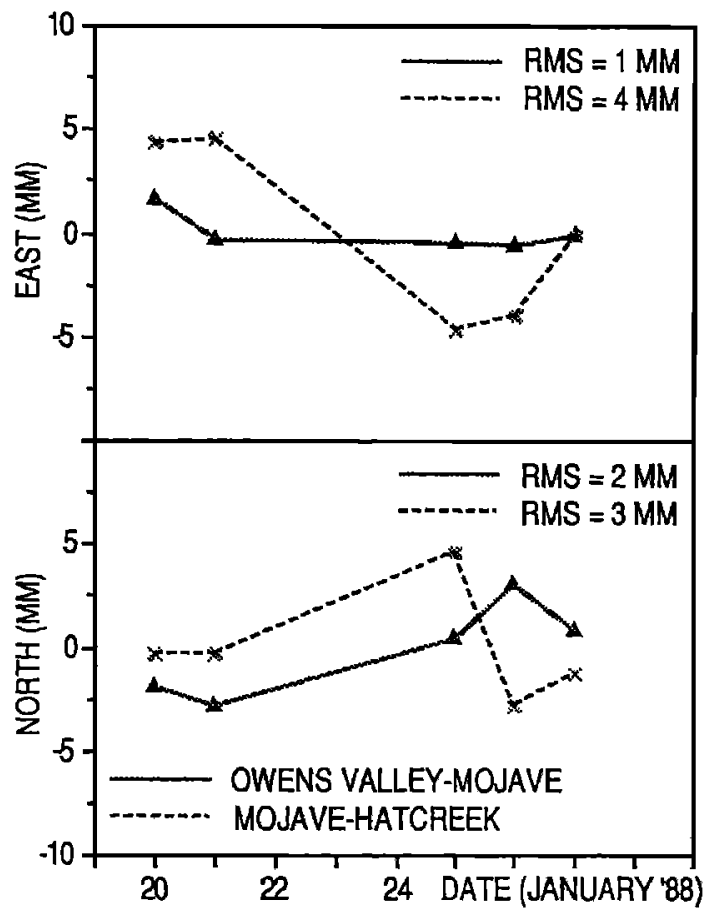

Fig. 3. Millimeter-level daily repeatabilities in horizontal baseline components with GPS for the Mojave-Hat Creek (729 $\mathrm{km})$ and the Owens Valley-Mojave $(245 \mathrm{~km})$ baselines. Results are plotted about their mean values and are based on single-day constrained orbit solutions.

The goal was to exhibit the precision possible with GPS under optimal conditions. Shown in figure 3 are horizontal daily repeatabilities for the Owens Valley-Mojave $(245 \mathrm{~km})$ and for the Mojave-Hat Creek (729 km) baselines. The horizontal rms scatter for the Owens Valley-Mojave baseline is $1-2 \mathrm{~mm}$, and for Mojave-Hat Creek it is 3-4 mm. The daily repeatability in the vertical components are $17 \mathrm{~mm}$ and $13 \mathrm{~mm}$ for the two baselines. Additional baselines studied included Owens Valley-Hat Creek (484 km), Hat Creek-Mammoth $(416 \mathrm{~km})$, and Mojave-Mammoth $(313 \mathrm{~km})$. When orbits are constrained all baselines produce horizontal repeatability of 2-6 mm and vertical repeatability of $10-15 \mathrm{~mm}$. With orbit constraints much smaller than $40 \mathrm{~m}$, systematic biases appeared in baseline

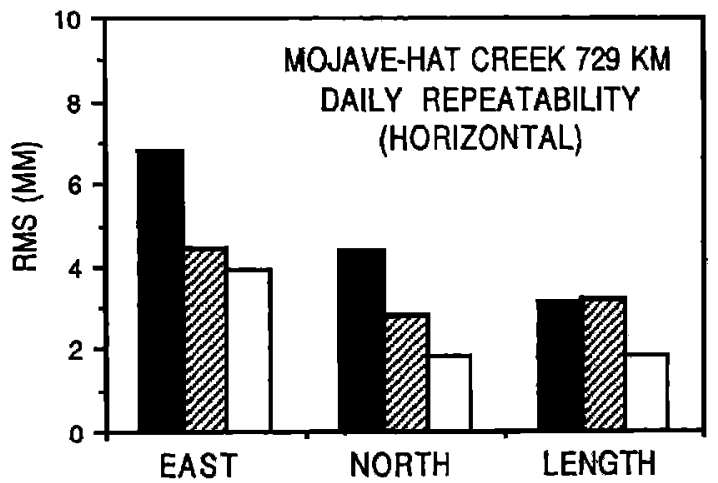

Fig. 4. Comparison of Mojave-Hat Creek (729 km) rms daily baseline repeatability for unconstrained (solid bars), constrained single-day (hatched bars), and multi-day (open bars) solutions. The two latter approaches yield baseline precision of $2-4 \mathrm{~mm}$ (about 3-5 parts in $10^{9}$ ), illustrating the advantages of weakly constraining the a priori orbit covariance for shont-arc estimation runs with a limited regional network. 
repeatabilities, but larger a priori sigmas $(50-80 \mathrm{~m})$, produced only slightly degraded results as using $40 \mathrm{~m}$ constraints.

The single-day unconstrained solutions yield $\mathrm{rms}$ errors of $3 \mathrm{~mm}$ in the horizontal and $19 \mathrm{~mm}$ in the vertical components for the Owens Valley-Mojave baseline and 4-7 mm horizontal and $22 \mathrm{~mm}$ vertical repeatabilities for the Mojave-Hat Creek baseline. In general the daily repeatabilities improve on average by a factor of $1.5,1.3$, and 1.2 for the east, north, and vertical components for the five baselines listed above, when a priori orbital information is used in parameter estimation. The assessment of GPS accuracy is difficult due to potential ground tie errors between GPS monuments and VLBI antenna intersection of axis. In addition, there is no VLBI solution for the site at Mammoth. For the constrained solution the agreement with VLBI remained between 10-20 $\mathrm{mm}$ in all components for the Owens Valley-Mojave and the Mojave-Hat Creek baselines. The Owens Valley-Hat Creek baseline agreed to $5-10 \mathrm{~mm}$ in the east and vertical components and differed by $36 \mathrm{~mm}$ in the north component. On average the agreement with VLBI improved relative to the unconstrained solution by a factor of $1.7,1.1$, and 1.7 for the east, north, and vertical components for the three baselines.

\section{Results From Multi-day Arc Solutions}

The multi-day arc strategy followed the one applied successfully to other experiments as described in Lichten and Bertiger (1989). All the single-day arc data and the same fiducials were used for the multi-day arcs plus data from four sites in South America and two sites in Europe. In the factorized Kalman filter stochastic zenith tropospheric delays and stochastic GPS solar radiation coefficients were estimated. The multi-day arc solutions were obtained with no a priori constraints applied to the orbital parameters. Formal orbit errors were at the meter-level or better for all components for the seven satellites tracked over much of the week-long data arc. The estimated Mojave-Hat Creek baseline agreed with VLBI to $10 \mathrm{~mm}$ or better and showed rms daily scatter of several millimeters in the horizontal and baseline length components (see figure 4). The agreement with VLBI is consistent with the sub meter formal errors in the GPS orbits. The multi-day arc orbit solution served as a reference for comparison with various single-day arc solution strategies.

In figure 4, daily repeatability for the Mojave-Hat Creek baseline is shown for the multi-day arc orbit case and for the single-day, constrained and unconstrained orbit cases. With the two former strategies, the horizontal and length precision is 3-5 parts in $10^{9}(2-4 \mathrm{~mm})$ and for the unconstrained case the precision is 6-9 parts in $10^{9}$ for the horizontal components and 4 parts in $10^{9}$ for the length component. For most baselines in this study the constrained single-day arc solutions performed nearly as well as the multi-day arc approach, even though European and South American sites were not included. In the vertical, both approaches had daily repeatability of $\sim 13 \mathrm{~mm}$ for Mojave-Hat Creek. Since the same fiducials were used, all three approaches produced essentially similar GPS-VLBI agreement $(10-20 \mathrm{~mm})$. For the considerably shorter $245 \mathrm{~km}$ Owens Valley-Mojave baseline, orbit error plays a less important role in the baseline error budget, and the horizontal single-day arc repeatabilities were 1-2 $\mathrm{mm}$ or better (as shown in figure 3), with no additional improvement when multi-day arc orbits were used. Clearly, the single-day constrained orbit strategy for the Owens Valley-Mojave baseline reduces orbit errors to a level which is lower than errors from other sources, such as multipath, troposphere mismodeling, and data noise.

\section{Discussion and Conclusions}

Our results indicate that few millimeter-level precision may be achievable in horizontal components of baselines up to 700 hundred $\mathrm{km}$ in length, using either constrained single-day solutions or multi-day arc computations, despite the handicaps of a limited constellation of GPS satellites and receivers with only four satellite channels. For the multi-day arcs the orbital state is better determined due to the repeated revolutions of the satellites. The use of a priori information to initially constrain the orbit covariances consistently improves solutions with weak observability, such as those from single-day arcs. The latter strategy is valid provided the applied constraints have not been determined from the same data set used for estimation.

Although the nominal orbit used for this study was based on broadcast ephemeris data, the conclusions should apply to nominal orbits produced by reduction of several days of GPS data taken from global tracking sites, e.g. Swift, 1985. This is especially pertinent, since the broadcast ephemeris will be significantly degraded by "selective availability" in the near future. Consequently, a practical scenario for future regional GPS analysis may involve the timely dissemination of precise ephemeris tables by a dedicated analysis group, which could be used in a similar fashion as the broadcast ephemeris data for producing a nominal orbit. Subsequently, the orbit, with a priori constraints, would be adjusted on a day by day basis using data from a user's regional network plus a three station, continental scale fiducial network which encompasses the region. The considerable savings in computational time and disk space makes this option attractive for users who do not wish to be burdened with the task of reducing many days of data from a worldwide set of GPS tracking stations.

Acknowledgments. The work described in this letter was carried out by the Jet Propulsion Laboratory, California Institute of Technology, under contract with the National Aeronautics and Space Administration.

\section{References}

Blewitt, G., Carrier phase ambiguity resolution for the global positioning system applied to geodetic baselines up to 2000 km, J. Geophysical Res., 94, 10187-10203,1989.

Beutler, G., I. Bauersima, W. Gurtner, M. Rothacher, and T. Schildnecht, Evaluation of the 1984 Alaska positioning campaign with the Beamese GPS software, J.Geophysical Res., 22, 1295-1303, 1987.

Dong, D., and Y. Bock, GPS network analysis with phase ambiguity resolution applied to crustal deformation studies in California, L. Geophysical Res, 94, 3949-3966, 1989.

Lichten, S.M., and W.I. Bertiger, Demonstration of sub-meter GPS orbit determination and 1.5 parts in $10^{8}$ threedimensional baseline accuracy, Bull. Geod.663, 167, 1989.

Lichten, S.M., W.I. Bertiger, and U.J. Lindqwister, The effect of fiducial network strategy on high-accuracy GPS orbit and baseline determination, Proc. 5th Intl. Geoderic Symposium on Satellite Positioning, I, pp. 516-525, 1989.

Lichten, S.M., and J.S. Border, Strategies for high-precision global positioning system orbit determination, J Geophysical Res, $92,12751-12762,1987$.

Swift, E., NSWC's GPS orbit/clock determination system, Proc. First Int. Symp. on Precise Positioning with GPS, ed. Goad, Vol. I, p. 51-62, Rockville, MD, May 1985.

U. Lindqwister, S. Lichten, and G. Blewitt, MS: 238-624, Jet Propulsion Laboratory, California Institute of Technology, Pasadena, CA 91109.
(Received November 8, 1989; revised January 30, 1990; accepted February 1, 1990) 